\title{
DYNAMIC PRICE COMPETITION WITH NETWORK EFFECTS
}

\author{
Luís Cabral
}




\title{
Dynamic Price Competition with Network Effects
}

\author{
Luís Cabral* \\ IESE Business School and CEPR
}

This draft: December 2009

\begin{abstract}
I consider a dynamic model of competition between two proprietary networks. Consumers die and are replaced with a constant hazard rate; and firms compete for new consumers to join their network by offering network entry prices.

I derive a series of results pertaining to: (a) existence and uniqueness of symmetric equilibria, (b) monotonicity of the pricing function (e.g., larger networks set higher prices), (c) network size dynamics (increasing dominance vs. reversion to the mean), and (d) firm value (how it varies with network effects).

Finally, I apply my general framework to the study of termination charges in wireless telecommunications. I consider various forms of regulation and examine their impact on firm profits and market share dynamics.
\end{abstract}

${ }^{*}$ Academic Director (U.S.), IESE Business School; lcabral@iese.edu. I am grateful to Mike Riordan for extensive discussions on matters related to this paper. I also thank Ennio Stacchetti, Andrea Prat, four referees, and seminar participants at (in chronological order) Yale, NYU, Cornell, Cambridge, Oxford, LSE, Ohio State, Nova, Autoridade da Concorrência, SED Prague meetings, Sidney, Melbourne, New South Wales, ANU, Queensland, Iowa State, Vanderbilt, Drexel, IESE Business School, Navarra, Munich, Johns Hopkins, Edinburgh, Imperial College, LBS, Southampton, Toulouse, UAB, Warwick, East Anglia, Porto, Bologna, and the Econometric Society U.S. meetings for useful comments. Naturally, I am solely responsible for errors and omissions. 


\section{Introduction}

Many industries exhibit some form of network effects, the situation whereby a consumer's valuation is increasing in the number of other consumers buying the same product (that is, the number of consumers in the same "network"). The most obvious source of network effects is direct network effects. Take the example of operating systems. If I use the Windows OS then, when I travel, it is more likely I will find a computer that I can use (both in terms of knowing how to use it and in terms of being able to run files and programs I carry with me). ${ }^{1}$

A second source of network effects is the availability of complementary products. For example, it seems reasonable that the variety and quality of software available for the Palm system is greater the more users buy PDAs that run Palm OS. A similar argument applies for complementary services. For example, the greater the number of Canon photocopiers are sold, the more likely it is that I will be able to find good post-sale service providers.

Finally, a third source of network effects is the pricing of network services. $^{2}$ Take the example of wireless telecommunications. To the extent that operators set different on-net and off-net prices, the utility from being connected to a given network is increasing in the number of other users on the same network.

In this paper, I consider a dynamic model of competition between two proprietary networks. Consumers die and are replaced with a constant hazard rate. Firms compete for new consumers to join their network by offering network entry prices (which may be below cost). New consumers have a privately known preference for each network. Upon joining a network, in each period consumers enjoy a benefit which is increasing in network size during that period. Firms receive revenues from new consumers as well as from consumers already belonging to their network.

I develop a general model with the above features. I derive the firms' and the consumers' value functions, both of which are a function of current network sizes. I provide conditions such that there exists a unique Markov equilibrium. The key is that, differently from static models, in an overlap-

1. Another source of direct network effects would be file sharing. While this is frequently proposed as the main source of direct network effects, in the example at hand I think it is relatively less important.

2. Laffont, Rey and Tirole (1998a) refer to this case as "tariff-mediated network externalities." 
ping generations framework consumers effectively make their network choices sequentially.

I then characterize the equilibrium, using a combination of analytical and numerical methods. One set of results pertains to the pricing function. As is frequently the case with dynamic games, there are two effects to consider. Larger networks are more attractive to consumers. This implies that, ignoring the firms' future payoffs, larger networks should set higher prices. However, in terms of future payoffs, larger firms have more to gain from increasing their network size then smaller firms. This dynamic version of the "efficiency effect" (duopoly joint profits are greater the greater the asymmetry between firms) leads larger firms to price lower. I provide conditions such that each of these effects dominates.

Although the equilibrium is symmetric, both the birth and the death processes are stochastic. Consequently, the actual state of the system (each firm's network size) is generally asymmetric. I show that a larger network is generally more likely to attract a new consumer (weak market dominance). Moreover, if network effects are sufficiently strong, then the larger network tends to increase in size (strong market dominance), unless it holds close to $100 \%$ of the market, in which case it tends to decrease in size. As a result, when network effects are sufficiently strong the stationary distribution of market shares is typically bimodal - the system spends most of the time at states where the large network has a market share between 50 and 100 percent.

Finally, I apply my general framework to the study of termination charges in wireless telecommunications. I consider three stylized forms of regulation: (a) termination charges set at marginal cost level, (b) symmetric termination charges higher than marginal cost, and (c) asymmetric termination charges (higher for smaller networks). I derive both the short-run and the long-run effects of these different regimes.

- Related literature. Following seminal work by Katz and Shapiro (1985), the early literature on oligopoly with network effects focused on relatively simple, static models. ${ }^{3}$ Since then, the industrial organization literature developed in two directions. One strand attempts to empirically measure

3. Other important early work includes Farrell and Saloner (1985), who focus on consumer behavior, and Arthur (1989), who presents an infinite period model but assumes non-proprietary networks (and thus excludes strategic behavior). 
the size of network effects. ${ }^{4}$ Another strand investigates further implications of network effects in an oligopoly context. ${ }^{5}$ Despite important developments, most of this literature has followed a static, or finite period, approach. ${ }^{6}$

More recent work attempts to explicitly address the issue of dynamic competition between proprietary networks. Doganoglu (2003), Mitchell and Skrzypacz (2006), derive the Markov Perfect Equilibrium of an infinite period game where consumer's utility is an increasing function of past market shares. Markovich (2004), Markovich and Moenius (2004) develop computational models of industries with "hardware" and "software" components (very much like my paper). They assume consumers live for two periods and benefit from indirect network effects through the quality of products available. Doraszelski, Chen, and Harrington (2007) also develop a computational dynamic model. In many respects, their analysis goes beyond my paper: for example, they analyze compatibility decisions, which I don't. However, like Doganoglu (2003), Mitchell and Skrzypacz (2006), they assume consumer benefits are an increasing function of network size at the time of purchase (that is, consumers are not forward looking). In sum, all of these papers assume relatively simple behavior on the part of consumers: either consumers are short-lived, or they are myopic, or they are backward looking. ${ }^{7}$ By contrast, I assume that consumers live for potentially many periods (that is, die with a constant hazard rate), and make their decisions in a rational, forward looking way. My paper also differs from theirs in that I look at a different set of issues.

In this sense, the papers that come closest to mine are Fudenberg and Tirole (2000), Driskill (2007), Laussel and Resende (2007), and Zhu and Iansiti (2007), all of which consider forward looking consumers. The framework considered by Fudenberg and Tirole (2000) is very specific: two consumers, two consumer types, etc.; the number and type of questions that can be addressed with such a simple model is therefore limited. Fudenberg and Tirole (2000) show that, under certain conditions, there exists an equilibrium whereby an incumbent firm sets a lower price in the presence of network externalities with a view at preempting competition by a potential entrant.

4. See, for example, Gandal (1994), Goolsbee and Klenow (2002), Rysman (2004).

5. See, for example, Laffont, Rey and Tirole (1998a,b).

6. Farrell and Klemperer (2006) present an excellent survey of this literature. See also Economides (1996).

7. See also Kandori and Rob (1998), Auriol and Benaim (2000), who approach the problem from a stochastic evolutionary perspective. 
The issue of network effects and pricing is also present in Driskill (2007). He considers a deterministic, continuous-time model where consumers are forward looking. He shows, among other things, that steady-state prices are lower in the presence of network externalities. My framework differs from his in that I consider idiosyncratic consumer preferences, which generate stochastic dynamics: I show that prices may be higher or lower in the presence of network effects; I also explain the main forces leading to different price levels.

Laussel and Resende (2007) look at equilibria in linear Markov strategies. They show that, in equilibrium, network access prices are decreasing in firm size. I provide sufficient conditions such that this is the case; but I also provide sufficient conditions such that the opposite is true. Moreover, in my model equilibrium strategies are generally nonlinear.

Zhu and Iansiti (2007) look at the competition between an incumbent and an entrant platform. In some respects, their model is more complex than mine: for example, they explicitly consider investment decisions by developers of each platform (network). In other respects, their framework is simpler than mine: for example, they assume prices are fixed and exogenouly given. They make several interesting points. In particular, they show that, contrary to popular wisdom, indirect network effects may favor the entrant platform (see also Llobet and Manove, 2006). In my paper, networks effects tend to hurt the small firm. The difference with respect to Zhu and Iansiti (2007) is partly due to the fact that I do not consider investments in quality.

Following the seminal contributions by Gilbert and Newbery (1982) and Reinganum (1982), a series of papers have addressed the issue of persistence of firm dominance. Contributions to this literature include Budd, Harris and Vickers (1993), Cabral and Riordan (1994), Athey and Schmutzler (2001), Cabral (2002). These papers provide conditions under which larger firms tend to become larger (market dominance). Intuitively, the reason for such dynamics corresponds to some form of the "efficiency effect" characterized by Gilbert and Newbery (1982): the fact that joint profits are greater the closer the market structure is to monpoly. My framework and results are consistent with the idea of market dominance. Specifically, I show that, if network effects are sufficiently strong and the large firm is not too large, then market dominance holds.

From a methodological point of view, my framework has various similarities with Cabral and Riordan's (1994) study of dynamic competition with learning curves. In many respects, one can interpret learning curves as a "sequential network effect." In fact, some of Cabral and Riordan's (1994) 
results regarding market dynamics hold in the context of network effects: in particular, the idea that larger firms tend to become even larger. However, there are two important differences between my framework and Cabral and Riordan (1994). First, Cabral and Riordan (1994) assume that learning curves "bottom out" at some level of learning (and there is no forgetting). This implies that the model can be solved backwards, starting with the state at which both firms reached the bottom of their learning curves. This in turn greatly simplifies the problem of finding a unique equilibrium. My current framework, by contrast, has consumers dying and being born. There is no absorbing state, rather firm size follows an ergodic Markov chain. This is more realistic in the context of network effects, but it also makes the analysis more complicated. Second, and more important, in Cabral and Riordan (1994) consumers play a passive role: they simply pick the best value for the money in each period. By contract, under network effects, I need to explicitly model consumer expectations regarding the evolution of network sizes.

- Structure of the paper. The remainder of the paper is organized as follows. In Section 2, I lay down the basic analytical framework, including the consumer and the firm optimization problems. Section 3 includes the main analytical results characterizing equilibrium dynamics, with a special emphasis on the price function and market share dynamics. In Section 4, I apply my general framework to a particular problem: access charge regulation in wireless telecommunications. Section 5 concludes the paper.

\section{Model}

I consider an infinite period model of price competition between two proprietary networks, owned by firms $A$ and $B$. Since I analyze anonymous Markov equilibria, with some abuse of notation I denote each firm by the size of its network, $i$ or $j$. Network size evolves over time due to consumer birth and death. In each period, a consumer dies and a new consumer is born. The new consumer chooses between one of the existing networks and stays with 
it until death..$^{8,9}$

Specifically, the timing of moves in each period is summarized in Table 1. Initially, a total of $\eta-1$ consumers are distributed between the two firms, so $i+j=\eta-1$. A new consumer is born and firms simultaneously set prices $p(i), p(j)$ for the consumer to join their network. If the new consumer opts for network $i$, then firm $i$ receives a profit $p(i)$, whereas the consumer receives a one-time benefit from joining network $i, \zeta_{i}{ }^{10}$

After the new consumer makes his choice, there are a total of $\eta$ consumers divided between the two networks. During the remainder of the period, firm $i$ receives a payoff $\theta(i)$, whereas a consumer attached to network $i$ enjoys a benefit $\lambda(i)$. In others words, I treat network choice as a durable good, and assume there is some non-durable good attached to the durable good "network membership." I denote the market for the non-durable good as the aftermarket. Finally, at the end of the period one consumer dies, each with equal probability. ${ }^{11}$ In other words, a consumer from firm $i$ 's network dies with probability $i / \eta$.

Since my main goal is to understand the evolution of network size over time, I take the values $\theta(i)$ and $\lambda(i)$ as given, that is, I treat them as the reduced form of the stage game played in the aftermarket. I assume that $\lambda(i)$ and $\theta(i)$ are bounded. Some of the results in Section 3 assume further properties of $\theta(i)$ and $\lambda(i)$, in particular the following two:

Property 1 (increasing network benefits) $\lambda(i)$ is increasing.

8. In this sense, my framework is similar to that of Beggs and Klemperer (1992). They too consider a stationary number of consumers and assume that a newborn consumer, having chosen one of the sellers, sticks with it until death.

9. Although I work with a discrete time model, the underlying reality I have in mind is one of continuous time. Suppose that consumers die according to a Poisson process with arrival rate $\lambda$. Essentially, I consider the time between two consecutive deaths as a period in my discrete time model. By assuming risk-neutral agents, I can summarize the Poisson arrival process in a discount factor $\delta$ that reflects the average length of a discrete period: $\delta=\exp (-r / \lambda)$, where $r$ is the continuous time discount rate.

10. For simplicity, I assume zero cost. Alternatively, we can think of $p(i)$ as markup over marginal cost.

11. The assumption that each consumer dies with equal probability allows me to keep the state space one-dimensional. In the opposite extreme case when consumers live a fixed number of periods ( $\eta$, to keep total market size the same), the state space becomes $\eta$-dimensional. 
Table 1: Timing of model: events occurring in each period $t$.

\begin{tabular}{|l|c|c|}
\hline Event & $\begin{array}{c}\text { Value } \\
\text { functions }\end{array}$ & State of the game \\
\hline \hline Firms set network entry prices $p(i)$ & $v(i)$ & $i \in\{0, \ldots, \eta-1\}$ \\
\cline { 1 - 2 } $\begin{array}{l}\text { Nature chooses } \xi_{i}, \text { new consumer's } \\
\text { preference for network } i\end{array}$ & & \\
\cline { 1 - 2 } New consumer chooses network & $u(i)$ & $i \in\{0, \ldots, \eta\}$ \\
\cline { 1 - 2 } $\begin{array}{l}\text { Stage competition takes place: period } \\
\text { profits } \theta(i), \text { consumer surplus } \lambda(i)\end{array}$ & & \\
\hline One consumer dies (probability $\left.\frac{1}{\eta}\right)$ & & $i \in\{0, \ldots, \eta-1\}$ \\
\hline
\end{tabular}

Property 2 (increasing returns to network size) Both $\theta(i)$ and $\theta(i+$ 1) $-\theta(i)$ are increasing.

Property 1 is straightforward: the greater a network size, the greater each consumer's aftermarket surplus (weakly). The first part of Property 2 is also fairly straightforward: larger networks make at least as much money in the aftermarket as smaller networks. With regards to the second part of Property 2, note that increasing $\theta(i)$ first differences implies that

$$
\frac{\theta(i+1)-\theta(0)}{i+1} \geq \frac{\theta(i)-\theta(0)}{i}
$$

It thus implies that firms (weakly) enjoy network benefits, in the sense that aftermarket variable profit per consumer is nondecreasing in network size, that is, network benefits imply increasing returns to scale for firms.

One can find many examples that feature increasing network benefits according to Properties 1 and $2 .{ }^{12}$ One can also find examples where one of the properties, or both, fail. In Section 4, I consider an application to wireless telecommunications networks where the aftermarket corresponds to cell phone usage. I show that, if termination charges are symmetric (network $\mathrm{A}$ pays network B the same charge that network B pays network A) and greater than marginal cost, then we have increasing network benefits and increasing

12. Two examples are: after sales service (e.g., photocopiers, printers, cameras); and handheld operating systems (e.g., Palm, PocketPC). 
returns to network size. If however access charges are very asymmetric, then Property 1 and Property 2 may fail.

My focus is on the firms' pricing decision and the consumer's network choice. Specifically, I consider equilibria in Markov pricing and network choice decisions. The state is defined by $i$, the size of firm $i$ 's network at the beginning of the period, that is, when firms set prices and the newborn consumer chooses one of the networks. ${ }^{13}$ I next derive the consumer's and the firm's decisions in a Markov equilibrium.

- Consumer choice. Each consumer's utility is given by two components: $\zeta_{i}$ and $\lambda(i)$. The first component is the consumer's idiosyncratic preference for firm $i$, which I assume depends on the identity of firm $i$ but not on the size of its network (thus the use of a subscript rather than an argument). The value of $\zeta_{i}$ is also the consumer's private information. The second component is network benefit from a network with size $i$ (including the consumer in question), which I assume is independent of the firm's identity. I assume that consumers receive the $\zeta_{i}$ component the moment they join a network, whereas $\lambda(i)$ is received each period that a consumer is still alive (and thus varies according to the size of the network during each future period). ${ }^{14}$

I assume that the values of $\zeta_{i}$ are sufficiently high so that a newborn consumer always chooses one of the available networks; that is, the outside good is always dominated. This is not an innocuous assumption, as I will discuss in Section 3; but it greatly simplifies the analysis. In particular, it allows me to concentrate on the value of $\xi_{i} \equiv \zeta_{i}-\zeta_{j}$, the consumer's idiosyncratic relative preference for firm $i$ 's network. Notice that $\xi_{j}=-\xi_{i}$. I assume that $\xi_{i}$ is distributed according to $\Phi(\xi)$ (density $\phi(\xi)$ ), which satisfies the following properties:

13. Note that, when consumers enjoy network benefits, there are a total of $\eta$ consumers, divided across the two networks. However, at the time that prices are set there are only $\eta-1$ consumers, so $i \in\{0, \ldots, \eta-1\}$ at that moment. The state and the firm value functions are defined at this moment (beginning of period).

14. The assumption that $\zeta_{i}$ is received at birth is not important. I could have the consumer receive $\zeta_{i}$ each period of his or her lifetime. However, this way of accounting for consumer utility simplifies the calculations. The important assumption is that all consumer heterogeneity is encapsulated in the value of $\zeta_{i}$, not in the recurring network benefit $\lambda(i)$. Abandoning this assumption would make the state space extremely large and render the analysis considerably more complex. 


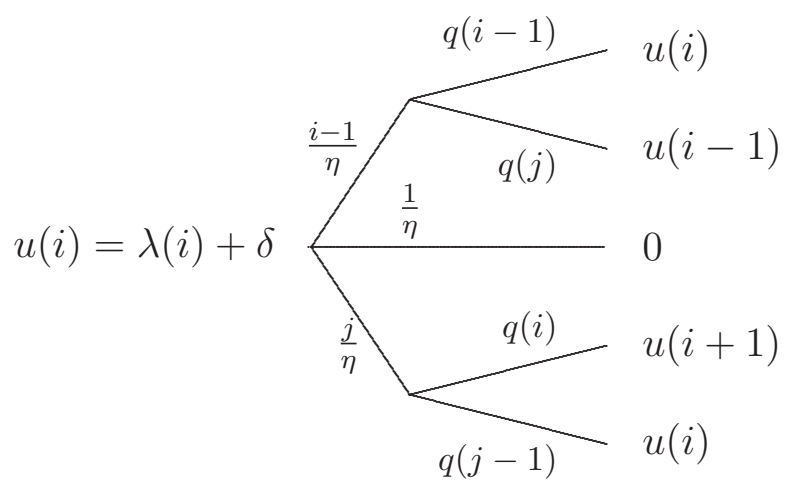

Figure 1: Consumer's value function.

Assumption 1 (i) $\Phi(\xi)$ is continuously differentiable; (ii) $\phi(\xi)=\phi(-\xi)$; (iii) $\phi(\xi)>0, \forall \xi ;(i v) \Phi(\xi) / \phi(\xi)$ is strictly increasing.

Many common distributions, including the Normal, satisfy Assumption 1.

Let $u(i)$ be a consumer's aftermarket value function, that is, the discounted value of payoff streams $\lambda(i)$ received while the consumer is alive (thus excluding both $\zeta_{i}$ and the price paid to join the network). Unlike the firm value functions, which I will measure at the beginning of each period, I will measure $u(i)$ after the current consumer has made his decision. This means that the argument of $u(i)$ varies from 1 to $\eta$.

Consider a new consumer's decision. At state $i$, the indifferent consumer will have $\xi_{i}=x(i)$, where the latter is given by

$$
x(i)-p(i)+u(i+1)=-p(j)+u(j+1),
$$

or simply

$$
x(i)=p(i)-p(j)-u(i+1)+u(j+1) .
$$

where $p(i)$ is firm $i$ 's price. This looks very much like a Hotelling consumer decision, except for the fact that $u(i+1)$ and $u(j+1)$ are endogenous values.

Firm $i$ 's demand is the probability of attracting the new consumer to its network. It is given by

$$
\begin{aligned}
q(i) & =1-\Phi(x(i)) \\
& =1-\Phi(p(i)-p(j)-u(i+1)+u(j+1)) .
\end{aligned}
$$


The consumer value functions, introduced above, are illustrated in Figure 1. The corresponding formula is given by

$$
\begin{gathered}
u(i)=\lambda(i)+\delta\left(\frac{j}{\eta} q(i) u(i+1)+\left(\frac{j}{\eta} q(j-1)+\frac{i-1}{\eta} q(i-1)\right) u(i)+\right. \\
\left.+\frac{i-1}{\eta} q(j) u(i-1)\right)
\end{gathered}
$$

where $q(i)$ is given by $(3), i=1, \ldots, \eta$, and $j=\eta-i .^{15}$ In words, a consumer's value is given, to begin with, by the current aftermarket benefit $\lambda(i)$. In terms of future value, there are three possibilities: with probability $1 / \eta$, the consumer dies, in which case I assume continuation utility is zero. ${ }^{16}$ With probability $(i-1) / \eta$, a consumer from the same network dies. This loss is compensated by the newborn consumer joining network $i$, which happens with probability $q(i-1)$, in which case next period's aftermarket state reverts back to $i$. With probability $1-q(i-1)$, the new consumer opts for the rival network, in which case next period's aftermarket state drops to $i-1$. Finally, with probability $j / \eta$, a consumer from the rival network dies. This loss is compensated by the newborn consumer joining network $j$, which happens with probability $q(j-1)$, in which case next period's aftermarket state reverts back to $i$. With probability $1-q(j-1)=q(i)$, the new consumer opts for network $i$, in which case next period's aftermarket state increases to $i+1$.

- Firm's pricing decision. Firm $i$ 's value function is given by

$$
\begin{aligned}
v(i)=q(i)( & \left.(i)+\theta(i+1)+\delta \frac{j}{\eta} v(i+1)+\delta \frac{i+1}{\eta} v(i)\right) \\
& +(1-q(i))\left(\theta(i)+\delta \frac{j+1}{\eta} v(i)+\delta \frac{i}{\eta} v(i-1)\right),
\end{aligned}
$$

where $i=0, \ldots, \eta-1$ and $j=\eta-1-i .^{17}$ This is illustrated in Figure 2 .

15. Recall that the argument of $u$ includes the network adopter to whom the value function applies, thus $i$ must be strictly positive in order for the value function to apply. For the extreme values $i=1$ and $i=\eta$, (4) calls for values of $q(\cdot)$ and $u(\cdot)$ that are not defined. However, these values are multiplied by zero.

16. Alternatively, I can consider a constant continuation utility upon death.

17. Again, notice that, for the extreme case $i=0,(5)$ calls for values of $v(\cdot)$ which are not defined. However, these values are multiplied by zero. 


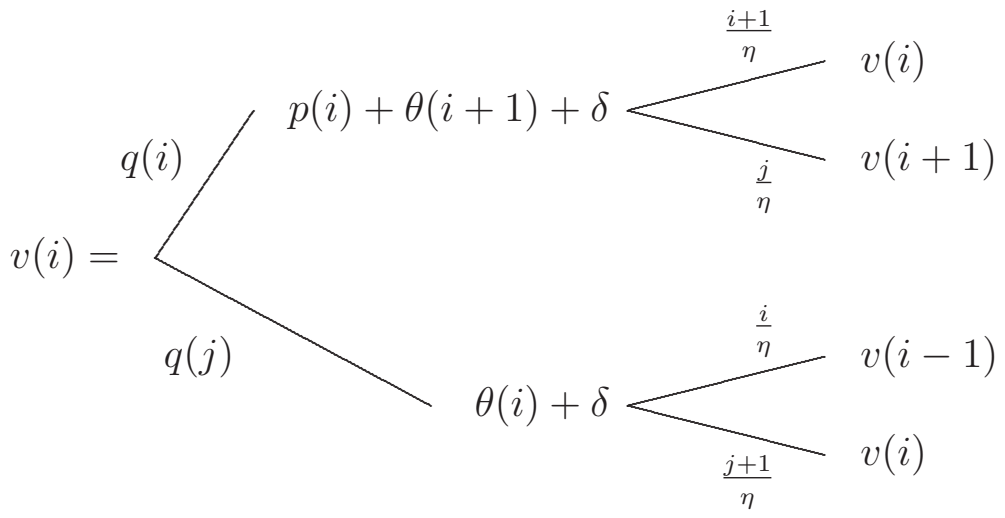

Figure 2: Firm's value function.

With probability $q(i)$, firm $i$ attracts the new consumer and receives $p(i)$. This moves the aftermarket state to $i+1$, yielding a period payoff of $\theta(i+1)$; following that, with probability $(i+1) / \eta$ network $i$ loses a consumer, in which case the state reverts back to $i$, whereas with probability $j / \eta$ network $j$ loses a consumer, in which case the state stays at $i+1$. With probability $q(j)$, the rival firm makes the current sale. Firm $i$ gets no revenues in the primary market. In the aftermarket, it gets $\theta(i)$ in the current period; following that, with probability $i / \eta$ network $i$ loses a consumer, in which case the state drops to $i-1$, whereas with probability $(j+1) / \eta$ network $j$ loses a consumer, in which case the state reverts back to $i$.

Equation (5), and the fact that $q(i)$ depends on $p(i)$ according to (3), leads to the following first-order conditions for firm value maximization:

$$
\begin{aligned}
q(i)+\frac{\partial q(i)}{\partial p(i)}( & p(i)+\theta(i+1)-\theta(i) \\
& +\delta \frac{j}{\eta} v(i+1)+\delta \frac{i+1}{\eta} v(i) \\
& \left.-\delta \frac{j+1}{\eta} v(i)-\delta \frac{i}{\eta} v(i-1)\right)=0
\end{aligned}
$$

or simply

$$
p(i)=h(i)-w(i)
$$


where

$$
\begin{aligned}
h(i) & \equiv \frac{q(i)}{-q^{\prime}(i)}=\frac{1-\Phi(x(i))}{\phi(x(i))} \\
w(i) & \equiv(\theta(i+1)-\theta(i))+\delta\left(\frac{j}{\eta} v(i+1)+\frac{i-j}{\eta} v(i)-\frac{i}{\eta} v(i-1)\right)
\end{aligned}
$$

In order to understand the intuition for the first order condition (6), it may help to rewrite it as follows:

$$
\frac{p(i)-(-w(i))}{p(i)}=\frac{q(i)}{-q^{\prime}(i) p(i)}=\frac{1}{\epsilon}
$$

where $\epsilon$ is the absolute value of the price elasticity of demand (by a newborn consumer). In other words, (7) is similar to the standard static profit maximization elasticity rule, with one difference: instead of the production marginal cost, which for simplicity I assume is zero, we have a negative "cost" of $-w(i)$. The value $w(i)$, is firm $i$ 's incremental future value from winning the current sale. By "future" I mean beginning with the current period's aftermarket. In terms of current period's payoff, the difference comes to $\theta(i+1)-\theta(i)$. In terms of future payoffs, we have the difference in value function between states $i+1$ and $i$ (if consumer death takes place in network $j$ ) or between states $i$ and $i-1$ (if consumer death takes place in network $i$ ).

With this comparison in mind, we can see that the first term on the right-hand side of (6) roughly corresponds to the standard markup under monopoly pricing. The only difference is that consumer demand includes the endogenous value difference $u(i+1)-u(j+1)$ : recall that the indifferent consumer "address" $x(i)$ is given by $x(i)=p(i)-p(j)-u(i+1)+u(j+1)$. The first term thus reflects the firm's "harvesting" price incentives (i.e., the lower the demand elasticity, the higher the price).

The second term reflects the firm's "investing" price incentives; that is, the more a firm has to gain, in terms of future payoffs, from making the current sale, the lower price it will set. I will return to this in the next section.

Finally, substituting (6) into (5) and simplifying, we get

$$
v(i)=r(i)+\theta(i)+\delta\left(\frac{j+1}{\eta} v(i)+\frac{i}{\eta} v(i-1)\right),
$$


where

$$
r(i) \equiv(1-\Phi(x(i))) h(i)=\frac{(1-\Phi(x(i)))^{2}}{\phi(x(i))} .
$$

This system is defined by a lower triangular matrix, and can thus be solved by forward substitution. The solution is given by

$$
v(i)=\left(1-\delta \frac{\eta-i}{\eta}\right)^{-1}\left(r(i)+\theta(i)+\delta \frac{i}{\eta} v(i-1)\right)
$$

$i=0, \ldots, \eta-1$. For $i=0$, we obtain $v(0)$ from $r(0)$ and $\theta(0)$. Then the value of $v(1)$ is given by $v(0)$ as well as $r(1)$ and $\theta(1)$; and so forth.

- Transition matrix and steady state distribution. Given the equilibrium values of $q(i)$, I can compute a Markov transition matrix $M=m(i, k)$ where $m(i, k)$ is the probability of moving from state $i$ to state $k$. For $0<i<\eta-1$, we have

$$
\begin{aligned}
m(i, i-1) & =\frac{i}{\eta}(1-q(i)) \\
m(i, i) & =\frac{i+1}{\eta} q(i)+\frac{\eta-i}{\eta}(1-q(i)) \\
m(i, i+1) & =\frac{\eta-1-i}{\eta} q(i)
\end{aligned}
$$

Moreover, $m(i, k)=0$ if $k<i-1$ or $k>i+1$. Finally, the boundary values are obtained as follows. For $i=0$, I apply the general equations and add the value obtained for $m(0,-1)$ to the value of $m(0,0)$. For $i=\eta-1$, again I apply the general equations and add the value obtained for $m(\eta-1, \eta)$ to $m(\eta-1, \eta-1)$. As a result, I get

$$
\begin{aligned}
m(0,0) & =1-\frac{\eta-1}{\eta} q(0) \\
m(0,1) & =\frac{\eta-1}{\eta} q(0) \\
m(\eta-1, \eta-2) & =\frac{\eta-1}{\eta}(1-q(\eta-1)) \\
m(\eta-1, \eta-1) & =1-\frac{\eta-1}{\eta}(1-q(\eta-1))
\end{aligned}
$$


Table 2: Notation.

\begin{aligned} & \hline$i$ Firm $i$ 's network size (also $j$ ) \\ &$\eta$ Market size (number of consumers). \\ &$\delta$ Discount factor. \\ &$\xi_{i}$ Consumers's idiosyncratic preference for firm $i \\ &. \Phi\left(\xi_{i}\right)$ Distribution of $\xi_{i}$. \\ &$\theta(i)$ Firm's aftermarket profit in state $i \\ &. \lambda(i)$ Consumer's aftermarket benefit in state $i \\ & x.(i)$ Indifferent consumer's relative preference for firm $i \\ & p.(i)$ Price in state $i$ (for new consumer). \\ &$q(i)$ Probability of a sale in state $i$ (to new consumer). \\ &$u(i)$ Individual consumer's value in state $i \\ & v.(i)$ Firm's value in state $i \\ & m.(i, j)$ Transition probability from state $i$ to state $j \\ & d.(i)$ Stationary probability density of state $i \\ &$. \hline\end{aligned}

Given the assumption that $\Phi(\cdot)$ has full support (part (iii) of Assumption 1$), q(i) \in(0,1) \forall i$, that is, there are no corner solutions in the pricing stage. It follows that the Markov process is ergodic and I can compute the stationary distribution over states. This is given by the (transposed) vector $d$ that solves $d M=d .^{18}$

A summary of the model's notation is given in Table 2. To help the reader navigate through the extensive set of variables, I follow the rule of using Greek letters to denote exogenous values and Roman letters to denote endogenous values.

\section{Results}

In this section, I characterize the equilibrium of the model introduced in the previous section. A preliminary question of interest is existence and uniqueness. Once that is established, I will be interested in (a) the price function, (b) the evolution of market shares, and (c) firm profits.

18. This vector $d$ can also be computed by repeatedly multiplying $M$ by itself. That is, $\lim _{k \rightarrow \infty} M^{k}$ is a matrix with $d$ in every row. 
Regarding the price function, the main question of interest is whether, under network effects, $p(i)$ is increasing or decreasing. The answer is not obvious. On the one hand, we might expect the large network to exploit its consumers' greater willingness to pay and charge a higher price. On the other hand, the large network may have more to lose from failing to sign up a new consumer, and thus price more aggressively than the small network.

Regarding the evolution of market shares, I define two concepts: weak and strong market dominance. We say there is weak market dominance when the large network attracts a new consumer with higher probability than the small network, that is, $q(i)>q(j)$ if and only if $i>j$. Weak market dominance does not imply that the large network tends to increase in size. In fact, since the death rate is given by $i / \eta$, the large network loses a consumer with greater probability than the small network. We then say there is strong market dominance when the large network increases its size in expected value, which happens if and only if $q(i)>i / \eta$. Since the large network's death rate is greater than the small network's, strong market dominance implies weak market dominance. ${ }^{19}$

Finally, regarding firm profits, the questions of interest are whether firm value is increasing in network size; and whether firm value and industry value are increasing or decreasing with the degree of network effects.

To recap, the model consists essentially of four sets of equations: the new consumer demand functions (3), the consumer value functions (4), the firm price functions (6), and the firm value functions (9). This system does not have a general analytical solution. As a result, I follow a two-pronged strategy. First, I derive analytical results (Propositions 1-5) for restricted sets of parameter values, specifically, low $\eta$, low $\delta$, and low $\psi$, where the latter parameter measures the degree of network effects. Second, I compute the

19. Athey and Schmutzler (2001) also distinguish between weak and strong market dominance. They propose an investment model of dynamic competition. In their definition, weak dominance means the leader invests more, whereas strong dominance means the leader increases its lead in expected terms. Although the mapping between my model and theirs is not exact, I believe my definition is the natural counterpart to theirs.

There is also an interesting parallel with the firm growth literature. Gibrat's law (growth rates are proportional to firm size) implies that the relative size of firms remains constant; it thus marks the threshold for strong market dominance. The threshold for weak market dominance, in turn, corresponds to Sutton's property that each new business opportunity is captured by incumbent firms with equal probability. I am grateful to Stephen Davies for pointing this out to me. 
solution numerically and determine the extent to which the limited analytical results extend to the rest of the parameter space.

Before getting into the main characterization results, I introduce three lemmas which provide a partial characterization of uniqueness and the shape of $q(i)$ and $p(i)$. These lemmas are useful in several ways. First, they help understand the intuition for equilibrium dynamics. Second, they are used in the proofs of propositions that will follow. Finally, the first lemma also forms the basis for the Gaussian method used in numerical computations. The lemmas (and the propositions that follow) make extensive use of differences across players. Specifically, I define

$$
\begin{aligned}
P(i) & =p(i)-p(j) \\
H(i) & =h(i)-h(j) \\
U(i) & =u(i+1)-u(j+1) \\
W(i) & =w(i)-w(j)
\end{aligned}
$$

In words, a capital Roman letter variable denotes the difference between player $i$ 's variable and player $j$ 's. The only exception to this rule, $U(i)$, takes into account the fact that, when a newborn consumer joins network $i$, that network's size increases to $i+1$.

Lemma 1 Given $\{U(i), W(i)\}$, there exist unique $\{p(i), q(i)\}$ satisfying equilibrium conditions (3) and (6); given $\{p(i), q(i)\}$, there exist unique $\{u(i), v(i)\}$ satisfying equilibrium conditions (4) and (9).

The proof of this and the following results may be found in the Appendix. Notice that $\{U(i), W(i)\}$ are uniquely determined by $\{u(i), v(i)\}$. Therefore, a corollary of Lemma 1 is that, given $\{u(i), v(i)\}$, there exist unique $\{p(i), q(i)\}$ satisfying equilibrium conditions (3) and (6). ${ }^{20}$

While Lemma 1 does not prove equilibrium uniqueness, it shows how uniqueness is obtained in my model when it is so difficult to obtain it in static models. Since consumers make their decisions in a staggered way (one at a time), a "small" consumer essentially takes future value functions as

20. Caplin and Nalebuff (1991) prove equilibrium uniqueness (Proposition 6) for a game similar to the static game obtained when $\delta=0$ or when $\{u(i), v(i)\}$ are given. Instead of my Assumption 1, they assume that $\phi$ is log-concave. Bagnoli and Bergstrom (2005) establish that my assumption is weaker than Caplin and Nalebuff's (1991). (I am grateful to a referee for the latter reference.) 
given. This means the problem of finding an equilibrium can be reduced to the firms' pricing and the newborn consumer's choice, which is essentially a garden-variety discrete choice problem.

Lemma 2 In equilibrium, the higher $U(i)+W(i)$ is, the higher $q(i)$ is. Moreover, $q(i) \geq \frac{1}{2}$ if and only if $U(i)+W(i) \geq 0$.

Lemma 2 implies that $q(i)>q(j)$ if and only if $U(i)+W(i)>0$. We thus have two sources of weak market dominance: $U(i)$ and $W(i)$. To the extent that consumers derive greater utility from a larger network, $U(i)>0$, which in turn increases the likelihood a new consumer joins the larger network. To the extent that a large network has more to gain from attracting a new consumer than a small network, $W(i)>0$. Since $p(i)=h(i)-w(i)$, the larger network, having a higher $w(i)$, prices more aggressively, which in turn increases the likelihood a new consumer joins the larger network.

An alternative way to understand the effect of $W(i)$ is to consider that, if $w(i)>w(j)$ when $i>j$, then industry continuation value is greater if the larger network makes the sale. That is, if we let $v^{+}$and $v^{-}$be continuation value with and without a sale, respectively, then $w(i)>w(j)$ is equivalent to $v^{+}(i)-v^{-}(i)>v^{+}(j)-v^{-}(j)$, which in turn is equivalent to $v^{+}(i)+v^{-}(j)>v^{-}(i)+v^{+}(j)$. In related research, Budd, Harris and Vickers (1993) and Cabral and Riordan (1994) also found that such joint-profit or joint-value effects lead to market dominance. Essentially, the joint-value effect corresponds to the dynamic version of Gilbert and Newbery's (1982) efficiency effect, whereby a monopolist has more to lose from not acquiring a new patent than a potential entrant has to gain from acquiring the same new patent.

I should note that these results depend on the assumption that the outside good is always dominated, that is, a newborn consumer always chooses one of the networks. If the outside good is not dominated, then I would need to consider a two-dimensional state space, keeping track of both $i$ and $j$. In economic terms, the no-outside-good assumption maximizes the preemption motives by the large firm. This is equivalent to the contrast between Gilbert and Newbery's (1982) result on persistence of monopoly and Reinganum's (1982) replacement counter-example. ${ }^{21}$

21. See Cabral (2000, Chapter 16) for a model that nests both Gilbert and Newbery (1982) and Reinganum (1982). 
Lemma 3 For a given $W(i)$, there exists a $U^{\prime}$ such that $P(i)>0$ if and only if $U(i)>U^{\prime}$. For a given $U(i)$, there exists a $W^{\prime}$ such that $P(i)<0$ if and only if $W(i)>W^{\prime}$.

The values of $U(i)$ and $W(i)$ measure the two sources of pricing incentives. To the extent that $U(i)$ is positive, consumers place a premium on network $i$. Everything else constant, this leads firm $i$ to set a higher price. We may refer to this as the "harvesting" effect. But everything else is not constant. A positive $W(i)$ implies that firm $i$ has more at stake in terms of future value. Everything else constant, this would lead firm $i$ to set a lower price. We may refer to this as the "investment" effect.

We thus have a "race" between harvesting and investing. Lemma 3 provides a (partial) characterization of prices with reference to this "race." It states that a larger network $i$ will set a higher price than a smaller network if and only if the harvesting effect, measured by $U(i)$, is sufficiently large with respect to the investment effect, measured by $W(i)$.

Equipped with these three lemmas, I now set out to characterize the solution for particular regions of the model's parameter set. I start with the case of low $\eta$.

Proposition 1 If $\eta=2$, then there exists a unique equilibrium. Moreover, if Properties 1 and 2 hold, then $q(1)>q(0)$ and $v(1)>v(0)$. Finally, there exists a $\lambda^{\prime}$ such that $p(1)>p(0)$ if and only if $\lambda(2)-\lambda(1)>\lambda^{\prime}$.

In the particular case when $\eta=2$, the values of $U(i)$ and $W(i)$ are completely determined by the primitives $\lambda(i)$ and $\theta(i)$. The first part of Proposition 1 then follows from Lemma 1. Moreover, Properties 1 and 2 imply that $U(i)>0$ and $W(i)>0$. The second part of Proposition 1 then follows from Lemma $2(q(1)>q(0))$ and simple algebraic manipulations $(v(1)>v(0))$. Finally, the result regarding prices follows from Lemma 3.

One question that might be asked about my framework is the role played by consumer rational expectations. Many of the existing models of network effects assume that consumers are short-lived, myopic, or naive. I define a naive consumer as one who assumes that network size will remain at its current level, that is, a consumer who fails to "solve" the model and correctly predict the evolution of network size. Denote by a tilde equilibrium variables corresponding to the case of naive consumers. Beginning with value functions, we have

$$
\tilde{u}(i)=\lambda(i)+\delta \tilde{u}(i)
$$


In words, a consumer in a network of size $i$ assumes that network size will remain the same. There are other ways of modeling naivete, but this seems a natural one. ${ }^{22}$

How do equilibrium values with naive consumers compare to the case of rational, forward-looking consumers? The next result provides the answer:

Proposition 2 Suppose that Property 1 holds. If $\eta=2$, then $\tilde{q}(0)<q(0)$ and $\tilde{v}(0)<v(0)$.

Intuitively, a small network suffers from consumer naivete because, in expected value, its network size can only increase - but consumers don't take that into account. For example, suppose that $\Phi(x)$ is a standardized Normal (consistent with Assumption 1); $\theta(0)=0, \theta(1)=\frac{1}{2}, \theta(2)=2, \lambda(1)=$ $\frac{1}{2}, \lambda(2)=1$ (all consistent with Properties 1 and 2); and $\delta=.9$. Then, in the equilibrium with rational consumers we have $q(0) \approx .199$, whereas with naive consumer we obtain $\tilde{q}(0) \approx .097$; that is, the model with naive consumers underestimates the probability of a sale by a small firm by less than one half. In terms of value, we have $v(0) \approx 7.96$ and $\tilde{v}(0) \approx 2.43$; that is, the model with naive consumers underestimates the value of a small firm by less than one third. Naturally, different parameter values lead to different estimate errors. However, my various simulations suggest that the prediction of Proposition 2 is not simply a theoretical possibility.

I next turn to the case when the discount factor, $\delta$, is small. I show uniqueness of equilibrium and characterize the price function (Proposition 3) as well as the evolution of market shares (Proposition 4).

Proposition 3 There exists a $\delta^{\prime}$ such that, if $\delta<\delta^{\prime}$, then there exists a unique equilibrium. Moreover, (a) if $\theta(i+1)-\theta(i)$ is constant and Property 1 holds strictly, then $p(i)$ is strictly increasing; $(b)$ if $\lambda(i)$ is constant and Property 2 holds strictly, then $p(i)$ is strictly decreasing.

Proposition 3 highlights the two main forces impacting on the firms' pricing incentives: market power over the current newborn consumer and the quest for market power in the aftermarket and in future periods. Analytically, we have $p(i)=h(i)-w(i)$, where the right-hand side represents the

22. For example, I could assume that the consumer allows for the possibility that he will die (which happens with probability $1 / \eta$ ). In that case, $\delta$ would be multiplied by $(\eta-1) / \eta$. The qualitative nature of Proposition 2 would remain valid. 
two effects on pricing. Proposition 3 considers the case when the discount factor is small. In this case, most of the effects are reflected in (current period's) aftermarket payoffs, which in turn allows me to derive conditions under which the first or the second effects dominate.

Specifically, in case (a) aftermarket profits are an affine function of network size. This implies that the benefit from winning a new customer, in terms of aftermarket profits, does not depend on network size: $w(i)$ is a constant. Differences in pricing are thus exclusively driven by market power considerations related to the newborn consumer, $h(i)$. Now, consumers are willing to pay more for a firm with a bigger network. In equilibrium, this is reflected in a higher price by the firm with a larger network. Thus $p(i)$ is increasing in $i$.

In case (b), consumers do not care about network size. (Notice this does not mean there are no network externalities, rather that sellers completely capture the added consumer surplus resulting from network externalities.) If it were not for aftermarket and future profits, firms would set the same price, as their products are identical in the eyes of consumers. But to the extent that $w(i) \approx \theta(i)-\theta(i-1)$ is increasing, the firm with a bigger network size has more to gain from making the next sale. This implies that it discounts price (with respect to the static price) to a greater extent. Thus $p(i)$ is decreasing in $i$.

Before presenting the next result, I define by $i^{*} \equiv \frac{\eta-1}{2}$ the "symmetric" state. If $\eta$ is even, then there exists no symmetric state, but the result below applies nonetheless. ${ }^{23}$

Proposition 4 Suppose that Properties 1 and 2 hold. There exist $\delta^{\prime}, \lambda^{\prime}, \theta^{\prime}$ such that, if $\delta<\delta^{\prime}$, then: (a) $q(i) \geq \frac{1}{2}$ if and only if $i>i^{*}$; (b) If $i$ is close to zero or close to $\eta-1$, then the state moves toward $i^{*}$ in expected terms; (c) If $i$ is close to $i^{*}$, and either $\lambda\left(i^{*}+1\right)-\lambda\left(i^{*}\right)>\lambda^{\prime}$ or $\theta\left(i^{*}+1\right)+\theta\left(i^{*}-1\right)-2 \theta\left(i^{*}\right)>$ $\theta^{\prime}$, then the state moves away from $i^{*}$ in expected terms.

In other words, Proposition 4 states that, if $\delta$ is small, then weak market dominance results. However, when market shares are close to zero or to $100 \%$, the death rate of a large network exceeds its birth rate, resulting in reversion to the mean (the opposite of strong market dominance). Around

23. Specifically, if $\eta$ is odd, then $i^{*}$ is the symmetric state. If $\eta$ is even, then there is no symmetric state; $i^{*}-\frac{1}{2}$ and $i^{*}+\frac{1}{2}$ are the two states closest to symmetry. 
symmetric states, if either $\theta(i)$ is very convex or $\lambda(i)$ very steep, then strong market dominance takes place.

To conclude the set of analytical results, I present a complete characterization of the equilibrium solution in the case when network effects are small, linear, and accrue to consumers in the form of aftermarket benefits.

Proposition 5 Suppose that $\lambda(i)=\psi i$ and $\theta(i)=0$. There exists a $\psi^{\prime}$ such that, if $\psi<\psi^{\prime}$, then: (a) Prices are increasing in network size; (b) Larger networks are more likely to attract a new consumer; (c) In expected terms, larger networks decrease in size; (d) Firm value is increasing in network size; (e) Industry profits are decreasing in the degree of network effects.

Parts (a), (b) and (c) of Proposition 5 are in line with Propositions 3 and 4. Parts (d) and (e) pertain to firm value in equilibrium. Together, they imply that, under network effects, having a larger network leads to greater firm value, but network effects uniformly decrease industry value. This is reminiscent of Theorem 3.5 in Cabral and Riordan's (1994) model of learning-by-doing. They show that, in equilibrium, industry value is lower than it would be in an environment where no learning-by-doing took place. In other words, while learning-by-doing and network effects increase total social value, they decrease total industry value. ${ }^{24}$ Notice that this result depends importantly on my assumption that there is no outside good (or equivalently that it is always dominated by the network goods). If there is a (relevant) outside good then network effects, by increasing the value of both network goods, are likely to imply an expansion in total market size, in which case industry value is more likely to increase.

- Numerical simulations. My theoretical results apply to extreme values of key parameter values $(\eta, \delta, \phi)$. In order to explore what happens for other parameter values, I performed a series of additional simulations. A detailed description of these simulations is available in an online appendix. The numerical results largely confirm the patterns described in the above analytical results, thus suggesting the latter do not depend on specific parameter values.

The numerical results also suggest patterns that are not obtained in the analytical results. Consider first the price function. At a theoretical level,

24. See Cabral and Villas-Boas (2005) for a generalization of this idea. 
I showed that there is a "race" between two effects, harvesting and investing. Numerical simulations show that, when after-market benefits accrue primarily to consumers, then we obtain an increasing price function (harvesting effect dominates); if, however, after-market benefits accrue primarily to firms, then we obtain a decreasing price function (investment effect dominates), unless $\delta$ is high and market share is high, in which case the price function is again positively sloped. Overall, these results are consistent with Proposition 3 (except for the case when $\delta$ is high).

Regarding market dominance, all numerical simulations confirm that $q(i)$ is increasing, thus extending the prediction of Propositions 1 and 4. Moreover, if network benefits are sufficiently strong, then strong market dominance takes place. Again, this is consistent with, and extends, the prediction of Propositions 1 and 4. Finally, one result that I do not derive analytically but is rather salient in the numerical simulations is that, as $\alpha$ changes from 0 to 1 , there is a shift towards greater market dominance.

\section{Application: wireless telecommunications}

In this section, I consider the application of my general framework to the study of wireless telecommunications. The application consists of adapting the static model of Laffont, Rey and Tirole (1998a,b) (hereafter LRT) to produce specific functional forms $\theta(i), \lambda(i)$. I then use the dynamic model developed in the previous sections to study the effects of regulatory policy regarding termination charges (also known as access charges).

In most developed countries, direct network effects play a relatively small role in telecommunications: by means of interconnection agreements, all callers are able to communicate with all other callers. However, if calls are priced differently depending on the calling parties' networks (typically with off-net calls being more expensive than on-net calls) then we have what LRT refer to as tariff-mediated network externalities (or network effects).

I assume that (as is the case in Europe) termination charges are set by regulators, and examine the implications of alternative policies. I show that, in addition to the immediate (or static) effect, one must also consider how different levels of termination charges lead to different dynamic paths of network market structure. Specifically, I show that higher markups over marginal cost, in addition to the short run deadweight loss characterized by LRT, imply a higher degree of market dominance, that is, a greater tendency 
for larger networks to become even larger.

- Model. Figure 3 illustrates the game played between networks in the aftermarket. This figure is essentially identical to Figure 1 in LRT, though the notation is slightly different. Each network sets the prices of on-net calls and off-net calls. For example, if $A$ calls $B$, who is in the same network, then $A$ is charged $\tilde{p}(i)$, the on-net call price for network $i$. If however $A$ calls $C$, who is in a different network, then $A$ is charged $\hat{p}(i)$, the off-net call price for network $i$. (I assume the receiving party is never charged.)

The main difference of my application with respect to LRT is that they assume all consumers simultaneous choose which network to join and how many calls to make. By contrast, I assume that, in each period, the size of each network is given. Only the newborn consumer decides which network it wants to join. All other consumers simply decide how many calls to make (given the network they belong to). ${ }^{25}$

In terms of costs, I follow LRT in assuming that the social cost of a call is given by $c_{0}+c_{1}+c_{0}$, where $c_{0}$ is the cost at each end of the call and $c_{1}$ is the trunk cost. Notice that cost is the same regardless of whether the call is on-net or off-net. In other words, like LRT I assume there are no "physical" benefits from network size.

For on-net calls (say, from $A$ to $B$ ), the cost $c_{0}+c_{1}+c_{0}$ is all borne by the network in question. For off-net calls (say, from $A$ to $C$ ), the calling network $\left(i\right.$, in this case) pays the cost $c_{0}+c_{1}$, corresponding to one end of the call and the trunk cost, plus the termination charge $a(j)$ which network $j$ requires for the service of terminating the call.

Suppose that, for each user pair, there is a utility of initiating calls given by

$$
u_{C}\left(q_{C}\right)=\left(\omega-\frac{1}{2} q_{C}\right) q_{C},
$$

where $q_{C}$ is the quantity of calls (or number of minutes) between the two users. In the appendix, I show this leads to the following consumer and firm aftermarket payoff functions:

25. Another difference with respect to LRT is that they consider both the case of linear prices (as I do) and two part tariffs. 


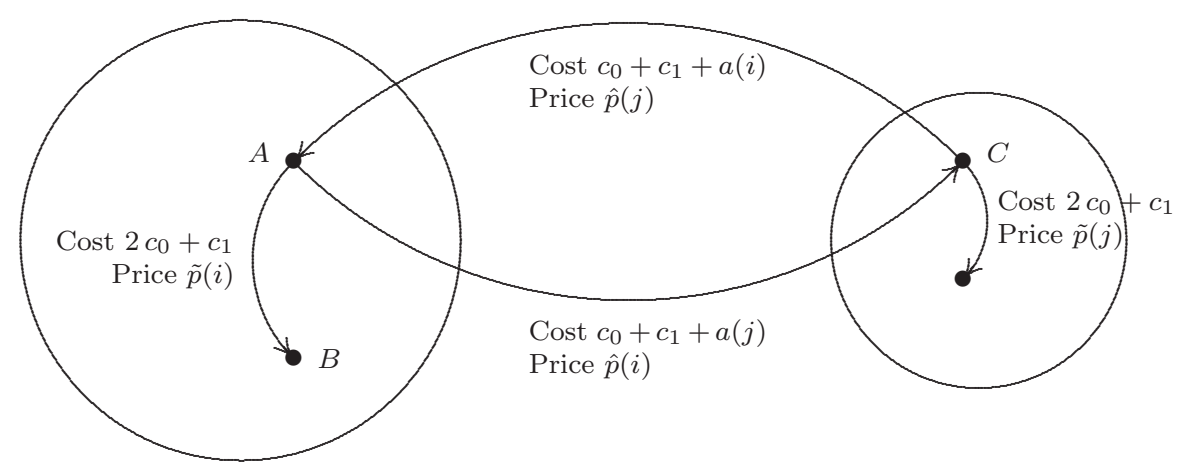

Network $i$ (size $i$ )

Network $j($ size $j)$

Figure 3: On-net and off-net calls.

$$
\begin{array}{r}
\lambda(i)=(i-1) \frac{1}{2}\left(\frac{\omega-2 c_{0}-c_{1}}{2}\right)^{2}+j \frac{1}{2}\left(\frac{\omega-c_{0}-c_{1}-a(j)}{2}\right)^{2} \\
\theta(i)=i(i-1)\left(\frac{\omega-2 c_{0}-c_{1}}{2}\right)^{2}+i j\left(\frac{\omega-c_{0}-c_{1}-a(j)}{2}\right)^{2}+ \\
+i j\left(\frac{\omega-c_{0}-c_{1}-a(i)}{2}\right)\left(a(i)-c_{0}\right)
\end{array}
$$

- Access charge and short-run profits. I now use my model to consider the static and dynamic implications of different values of the access charge $a(i)$ paid by network $j$ to network $i$. I consider three possible cases:

(A) Access charges are regulated at marginal cost level: $a(i)=c_{0}$.

(B) Access charges are set at twice marginal cost level: $a(i)=2 c_{0}$.

(C) Access charges are inversely related to network size, specifically,

$$
a(i)=\left(2-\frac{i}{\eta}\right) c_{0}
$$

In a certain sense, case $\mathrm{C}$ is a combination of cases $\mathrm{A}$ and $\mathrm{B}$ : a network of size zero charges $2 c_{0}$ (as in case $\mathrm{B}$ ), whereas a network of size $\eta$ charges $c_{0}$ (as in case A). 

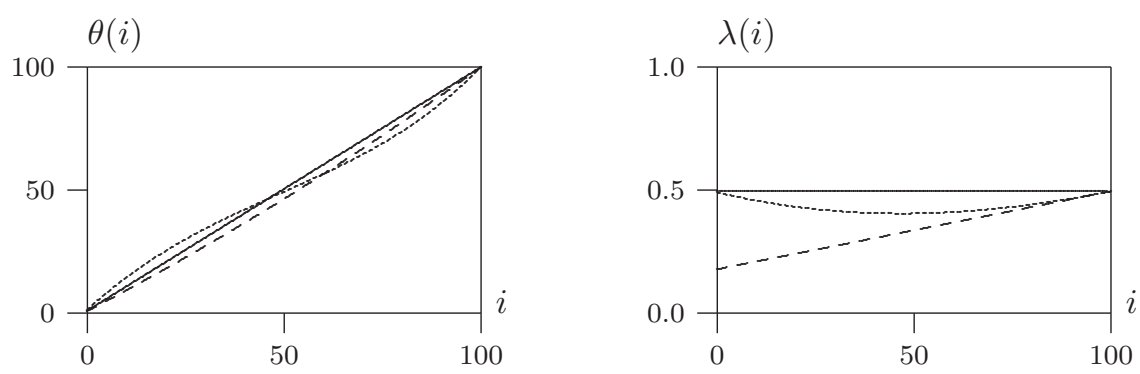

Figure 4: Firm profit and consumer surplus, under cases A (solid line), B (dashed line), and $\mathrm{C}$ (dotted line).

The above cases, while obviously very stylized, roughly reflect the policies of various European regulators in recent years. In particular, the consensus has been to converge to case A from the scenarios of recent years, which have been closer to B or C.

Figure 4 depicts firm profit and consumer surplus in the aftermarket as a function of market share. ${ }^{26}$ Consider first the left panel, firm profit. If access charges are set at marginal cost level (solid lines), then on-net and off-net calls cost the same. As a result, a firm's profit per period is simply proportional to its market share. If however firms set a fixed markup over cost, as in case B (dashed lines), then we have a situation of double marginalization (also characterized by LRT). This implies equilibrium profits below the case when access charges are set at marginal cost level. ${ }^{27}$ Finally, in case $\mathrm{C}$, the profit function $\theta(i)$, while still increasing, is now concave for low values of $i$ (thus violating Property 2) and convex for high values of $i$. Moreover, for low values of $i, \theta(i)$ is greatest in case C: in terms of current profits, small networks prefer asymmetric regulation, whereby they are able to charge a high termination charge while the rival (large) network is forced to charge a low one.

Consider now the right panel, which depicts consumer surplus as a func-

26. In all of the simulations presented in this section, I assume $\delta=.9, c_{0}=c_{1}=1$, $\omega=5$. Different parameter values lead to equivalent qualitative results.

27. Notice we are considering the case when both firms charge an access fee above cost. Given firm $j$ 's access fee, firm $i$ is better off by charging an access fee above cost. In other words, the one-shot game of setting access fees is a prisoner's dilemma: firms are better off with marginal cost pricing, but each firm has an incentive to set fees above marginal cost. 
tion of network size. Under marginal cost pricing (solid lines), consumer surplus is independent of network size: to the extent that on-net and off-net prices are the same, consumers do not care about the size of their network. Consider now case B, when termination charges are constant but greater than marginal cost. This leads networks to set higher off-net call prices than on-net call prices. As a result, consumer surplus is (linearly) increasing in $i$. Finally, consider case C. If network size is either equal to zero or to $\eta$, then consumer surplus is the same as under marginal cost pricing. If $i=0$, the rival network charges a termination charge that is equal to marginal cost and so, from a consumer's perspective, it is as if we were in case A. If $i=\eta$, then consumers do not care about access charges as none of their calls will be off-net anyway. For intermediate values of $i$, we observe that asymmetric regulation of termination charges may lead to a non-monotonic consumer surplus function (thus violating Property 1).

To summarize, setting termination charges above marginal cost implies a short-run economic loss which generally leads to a loss in firm profits and consumer surplus. Under asymmetric regulation, however, small networks are better off. Finally, notice that, while Properties 1 and 2 hold in cases A and $\mathrm{B}$, they both fail in case $\mathrm{C}$.

- Access charge and dynamic equilibrium. The short-run implications of markups in access charges are well known from LRT and other papers. My main point in the present application is that different regulatory regimes also have important dynamic implications. This is illustrated in Figure 5, where I plot the values of of various endogenous variables under each of the three scenarios I have been considering.

Consider first the top left-hand panel, $p(i)$, which depicts the price paid by a newborn consumer in order to join a network of size $i$. Under marginal cost access pricing, this price is independent of network size. In fact, to the extent that profits are proportional to market share, a firm's incentive to attract a new consumer is independent of its network size; and moreover consumer do not care about network size either. If however $a(i)=\bar{a}>c_{0}$, then two things happen: First, consumers are more willing to join a larger network (everything else constant). Second, firm $i$ 's payoff per period is a convex function of its market share. Together, these facts lead to a U shaped price function: for low values of $i$, the investment price incentive is very low (given convexity of the $v(i)$ ), leading to high prices; whereas for high values of $i$, the harvesting effect dominates (given an increasing $u(i)$ ), leading again to 

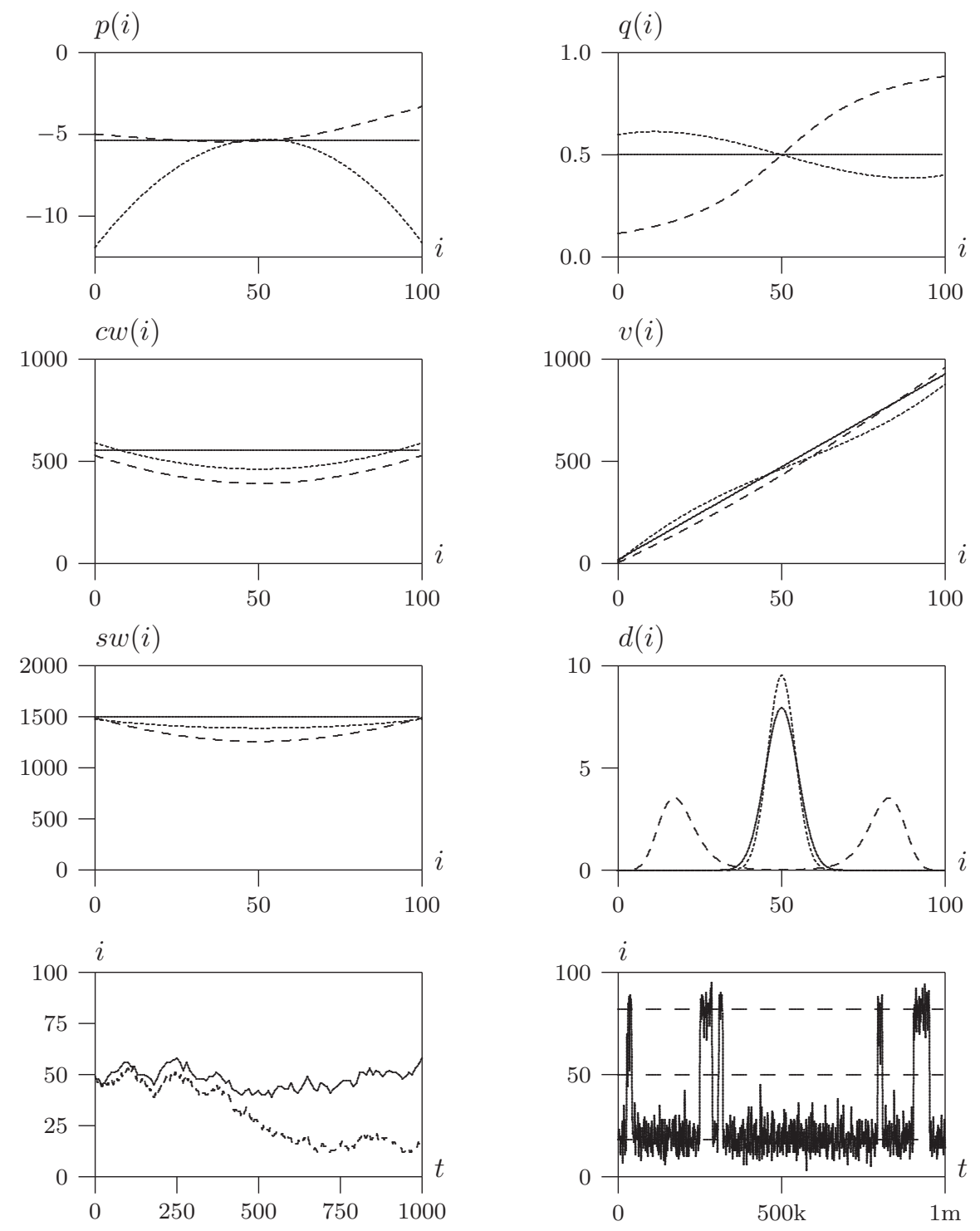

Figure 5: Representative results of access charge regulation simulations: Cases A (solid line), B (dashed line), and C (dotted line). 
high prices. (Strategic complementarity between firm prices is also a factor here.) This is broadly consistent with the analytical and numerical results presented in Section 3.

Finally, consider the case of asymmetric regulation. As mentioned earlier, this case implies that the derivative of firm profit with respect to network size is very large either for very low or for very high $i$. The pattern of the short run $\theta(i)$ function is reflected in the value function $v(i)$, as can be seen from the second right-hand panel. This leads firms to compete very aggressively for new consumers when market shares are very asymmetric. As a result, the pricing function has an inverted- $U$ shape. This is very different from the results obtained in Section 3, but recall that then I assumed Properties 1 and 2 held, whereas under case $\mathrm{C}$ these properties are violated.

Consider now the top right-hand panel, $q(i)$. Under marginal cost termination charges, consumers are indifferent regarding network size. This leads to a constant $50 \%$ probability of joining network $i$. Under a constant markup, however, larger networks become more attractive to consumers. Consistently with the results in Section 3, this leads to an increasing $q(i)$ function. In fact, if the markup over marginal costs is sufficiently high (as is the case with $\left.a(i)=2 c_{0}\right)$, then the slope of $q(i)$ is greater than $1 / \eta$ at $i=\eta / 2$. Finally, under scenario $\mathrm{C}$ we obtain a non-monotonic $q(i)$ mapping. This is different from the theoretical results in Section 3, where I assume Properties 1 and 2 hold. These properties are violated under Scenario 3. In particular, consumer benefit is decreasing in network size for small $i$.

Different mappings $q(i)$ lead to different stationary distributions over market shares. Under Scenarios A and C, the birthrate $q(i)$ is greater than the death rate if and only if $i<\eta / 2$. As a result, the dynamics are characterized by reversion to the mean and the stationary distribution of markets shares in unimodal. Under Scenario $\mathrm{B}$, for intermediate values of $i$ the birth rate is greater than the death rate if and only if $i>\eta / 2$. As a result, the dynamics are characterized by strong market dominance. This is illustrated in the third right-hand panel of Figure $5, d(i)$.

The two bottom panels illustrate the dynamics implied by the stationary distributions. The bottom left panel simulates Scenarios A and B for 1000 periods (Scenario C implies a similar pattern to Scenario A). Starting from the symmetric state, $i=i^{*}=50$, the system remains around $i^{*}$ most of the time under Scenario A but rapidly converges to one of the asymmetric states under Scenario B ( $i=18$ in the particular example). Under Scenario B, the system hovers around the asymmetric state for a "long" time. However, if we 
simulate the system for long enough, then "tipping" takes place, that is, the system moves across asymmetric states. This is illustrated by the bottom right panel, where I simulate the system under Scenario B for one million periods instead of one thousand.

I next turn to profits and welfare. The third left panel from the top, $s w(i)$ shows that social welfare is lower the higher the markup of termination charges over marginal cost: lowest in Case B, highest in Case A. Not surprisingly, the welfare loss is greater at intermediate states. In fact, at $i=0$ there are no off-net calls, and so all cases lead to the same level of social welfare. Notice that the panel only gives welfare as a function of the state. Average long-run welfare is then given by the "integral" of the $s w(i)$ mapping weighted by the stationary distribution over states. This distinction is important. For example, the simple average difference between Scenarios $\mathrm{B}$ and $\mathrm{C}$, in terms of social welfare, is favorable to the latter by about $5.65 \%$. However, when weighted by long-run probabilities, it is considerably lower, $2.57 \%$. In fact, it is theoretically conceivable that the two averages have different sign.

While social welfare is uniformly lower with termination charges markups, the different policies also have significant distributional implications (consumers vs firms). This is illustrated by the second row of panels in Figure 5. The left panel, $c w(i)$, shows that for $i$ close to zero (or close to $\eta$ ) consumers are better off under scenario $\mathrm{C}$. This is because firms compete vigorously for a new consumer; and, from the point of view of termination charges, consumers are nearly indifferent because there are very few off-net calls $(i \approx \eta)$ or the rival network charges access rates close to marginal cost $(i \approx 0)$. However, notice that, in the long run, these states are visited very infrequently.

From the right-hand panel, $v(i)$, we see that there is a range of low values of $i$ such that a firm is better off under Scenario $\mathrm{C}$ than Scenario A. It should be noted, however, that $v(0)$ is actually lower under Scenario $\mathrm{C}$ than under Scenario A. The difference is small and can hardly be noticed in Figure 5, but the idea should be clear. From a short-run aftermarket profit point of view, Scenarios A and $\mathrm{C}$ are not very different: a small network cares little about charging high termination charges since there are very few calls to terminate. However, Scenario $\mathrm{C}$ has an immediate negative effect: it increases competition for new customers, as shown in the top left panel. In addition to Scenario C, firm value at $i=0$ is also lower under Scenario B than under Scenario A. 
- Policy implications: protecting entrants. One of the salient features of my dynamic framework is that markups in access charges decrease the value of a firm with zero network (an "entrant"). This holds true regardless of whether termination charges are symmetric or asymmetric. My conclusion for the symmetric case is consistent with various static models, including those by Calzada and Valletti (2007) and Hoernig (2007). Peitz (2005), who considers a static model of network competition, shows that the small network's profit increases if the regulator sets a higher price to access the small network (asymmetric termination charge regulation). Consistently with his conclusion, my model predicts that the small network's profit in the after market is greater under asymmetric regulation. However, differently from the static model, the small network's value is lower under asymmetric regulation, because of increased competition for new consumers.

The discussion of the merits of asymmetric termination charge regulation is not of purely academic interest. Consider for example the following quote from a recent Common Position by the European Regulation Group (ERG):

Under some circumstances asymmetric mobile termination rates may be justified for example ... to encourage the growth of a new entrant on the market, which suffers from a lack of scale due to late market entry (European Regulation Group, 2008, p. 82)

In its contribution to the ERG consultation, and referring to asymmetric termination charge regulation, telecommunications operator Vodafone affirmed that

Asymmetries for 'late entrants' are entirely subjective and have no justification at all. Worse, they discourage later entrants from growing. (Vodafone, 2008, p. 2)

My analysis suggests that, differently from the ERG's claim, it is not necessarily the case that asymmetric regulation increases short-run profits of small operators or strengthens their relative competitive position (unless we interpret the latter as fast market share growth). Moreover, while my analysis agrees with Vodafone that asymmetric regulation may not help small operators, the reason is rather different: the value upon entry is lower, but conditional on entry growth takes place faster (because small operators have so much to gain from increasing market share). 
Admittedly, my model is very stylized; for example, I only consider two operators. However, it suggests that competition dynamics leads to a variety of effects that a static analysis may miss.

Summary. There is now an extensive literature dealing with the problem of wireless network competition and regulation of termination charges. ${ }^{28}$ To the best of my knowledge, all of the models developed are static in nature. The model I present in this section, in addition to illustrating my general framework, contributes to the literature in several ways. First, I show that, in addition to the static efficiency effects of different regulation modes, one must also consider the dynamic market share effects. Specifically, higher termination charges, by inducing tariff mediated network effects, lead to more dispersed market shares, possibly a bi-modal stationary distribution. Second, allowing small networks to charge higher termination charges, while increasing their short-run profits, may actually lead to lower firm value because of the more aggressive behavior by the large "incumbent" network.

\section{Final remarks}

In this paper, I propose a novel framework with which to analyze the dynamics of price competition with network effects. I provide a series of analytical results which characterize equilibrium dynamics: conditions for equilibrium existence and uniqueness; conditions such that the price function is increasing (larger firms set higher prices); and conditions such that market share dynamics are characterized by weak or strong market dominance. The numerical solution of the model suggests that the properties uncovered for extreme parameter values (low $\eta$, low $\delta$, low $\psi$ ) largely extend to other regions of the parameter space.

I then apply my framework to the study of termination charges (or access charges) in wireless telecommunications. I do so by embedding the Laffont, Rey and Tirole (1998a,b) model of competition between networks into my dynamic framework. I consider various forms of termination charge regulation and solve for the resulting equilibrium. The results suggest that, in addition to the static effects uncovered in previous research, there are interesting dynamic effects resulting from different regulatory regimes. For example, high markups of termination charges over marginal cost imply greater

28. For a survey, see Harbord and Pagnozzi (2008). 
market dominance, and possibly the switch from a uni-modal to a bi-modal stationary distribution of market shares.

There are other interesting issues that one can analyze with the framework presented in this paper. One is to estimate the barrier to entry created by network effects: how does the value of a new entrant depend on the degree of network effects? A second issue is innovation incentives under network effects: do larger networks have a greater incentive to improve their product than smaller networks? ${ }^{29}$

29. In order to answer this question, one would need to extend the present framework to the case of asymmetric firms. This is notationally painful; and increases the state space from $\eta / 2$ to $\eta$ states; but otherwise it is feasible. 


\section{Appendix}

Proof of Lemma 1: Subtracting the first-order conditions, I have

$$
P(i)=H(i)-W(i) .
$$

From (7), we have

$$
H(i)=\frac{1-\Phi(x(i))}{\phi(x(i))}-\frac{1-\Phi(x(j))}{\phi(x(j))}=\frac{1-2 \Phi(x(i))}{\phi(x(i))}
$$

since $x(j)=-x(i), \Phi(-x)=1-\Phi(x)$ and $\phi(-x)=\phi(x)$. Given that $x(i)=P(i)-U(i)$, equation (12) may then be re-written as

$$
P(i)+\frac{2 \Phi(P(i)-U(i))-1}{\phi(P(i)-U(i))}=-W(i)
$$

Given $U(i)$ and $W(i)$, the above equation has only one unknown, $P(i)$.

Note that, by part (ii) of Assumption 1,

$$
\frac{2 \Phi(x)-1}{\phi(x)}=\frac{\Phi(x)}{\phi(x)}-\frac{\Phi(-x)}{\phi(-x)}
$$

Part (iv) of Assumption 1 then implies that the left-hand side of (14) is increasing in $x$. It follows that, given $U(i)$, the left-hand side of (13) is an increasing function of $P(i)$, ranging from $-\infty$ to $+\infty$. The intermediate value theorem implies that there exists a unique equilibrium value $P(i)$. Given $\{P(i)\}$ (as well as $\{U(i)\}$ ), the values of $q(i)$ and $p(i)$ are uniquely determined by (3) and (6). (By $\{P(i)\}$ I mean the set of values $P(i)$ for all $i$.)

The reverse is straightforward. In fact, for given $\{p(i)\}$ and $\{q(i)\}$, (4) defines a linear system in $\{u(i)\}$; and (9) defines a linear system in $\{v(i)\}$; and $\{U(i)\}$ and $\{W(i)\}$ are uniquely determined by $\{u(i)\}$ and $\{v(i)\}$.

Proof of Lemma 2: Equation (12) may be re-written as

$$
P(i)-U(i)+H(i)=-(U(i)+W(i))
$$

or simply

$$
x(i)+\frac{2 \Phi(x(i))-1}{\phi(x(i))}=-(U(i)+W(i))
$$


since $x(i)=P(i)-U(i) \cdot{ }^{30}$

The left-hand side is increasing in $x(i)$ and is equal to zero if $x(i)=0$. It follows that $x(i)$ is decreasing in $U(i)+W(i)$ and $x(i) \leq 0$ if and only if $U(i)+W(i) \geq 0$. Finally, the result follows from the equation for $q(i)$, namely $q(i)=1-\Phi(x(i))$.

Proof of Lemma 3: Equation (13) may be rewritten as

$$
G(P(i))=-W(i)
$$

where

$$
G(P(i)) \equiv \frac{2 \Phi(P(i)-U(i))-1}{\phi(P(i)-U(i))}
$$

is an increasing function of $P(i)$. It follows that $P(i)>0$ if and only if $G(0)<-W(i)$. Since

we conclude that $P(i)>0$ if and only if

$$
\frac{2 \Phi(-U(i))-1}{\phi(P-U(i))}<-W(i)
$$

or simply

$$
\frac{2 \Phi(U(i))-1}{\phi(U(i))}>W(i)
$$

where I use the facts, implied by part (ii) of Assumption 1, that $\Phi(-x)=$ $1-\Phi(x)$ and $\phi(-x)=-\phi(x)$. Since the left-hand side is increasing in $U(i)$, the result follows.

Proof of Proposition 1: If $\eta=2$, then the consumer value functions (4) become

$$
\begin{aligned}
& u(1)=\lambda(1)+\delta \frac{1}{2}(q(1) u(2)+q(0) u(1)) \\
& u(2)=\lambda(2)+\delta \frac{1}{2}(q(1) u(2)+q(0) u(1))
\end{aligned}
$$

30. I am grateful to a referee for suggesting this approach. 
It follows that $U(1)=\lambda(2)-\lambda(1)$ is a constant (and so is $U(0)=-U(1)$ ).

The firm value functions (7) become

$$
\begin{aligned}
& w(0)=\theta(1)-\theta(0)+\delta \frac{1}{2}(v(1)-v(0)) \\
& w(1)=\theta(2)-\theta(1)+\delta \frac{1}{2}(v(1)-v(0))
\end{aligned}
$$

It follows that $W(1)=\theta(2)-2 \theta(1)+\theta(0)$ is a constant (and so is $W(0)=$ $-W(1))$.

Since $\{U(i), W(i)\}$ and all constants, Lemma 1 implies that there exist unique $\{p(i), q(i)\}$, satisfying the equilibrium conditions; and $\{u(i), v(i)\}$ are also uniquely determined by the equilibrium conditions. Moreover, Properties 1 and 2 imply that $U(1)+W(1)>0$, which in turn implies $q(1)>q(0)$, by Lemma 2 .

If $\eta=2$, then the value functions (8) become

$$
\begin{aligned}
& v(0)=r(0)+\theta(0)+\delta v(0) \\
& v(1)=r(1)+\theta(1)+\delta\left(\frac{1}{2} v(1)+\frac{1}{2} v(0)\right)
\end{aligned}
$$

Subtracting the first equation from the second one, we get

$$
v(1)-v(0)=(r(1)-r(0))+(\theta(1)-\theta(0))+\frac{\delta}{2}(v(1)-v(0))
$$

Since $q(1)>q(0)$, we have $r(1)>r(0)$. Property 2 implies $\theta(1)>\theta(0)$. It follows that $v(1)>v(0)$.

Finally, the result regarding prices follows from Lemma 3 and the fact that $U(1)=\lambda(2)-\lambda(1)$ and $W(1)$ is a constant.

Proof of Proposition 2: First notice that uniqueness of equilibrium follows by the same argument as when consumers are rational. From the proof of Proposition 1, we know that $U(1)=\lambda(2)-\lambda(1)$. Property 1 implies $U(1)>0$. From (11), we have $\tilde{u}(i)=(1-\delta)^{-1} \lambda(i)$, and thus

$$
\tilde{U}(1)=(1-\delta)^{-1}(\lambda(2)-\lambda(1))=(1-\delta)^{-1} U(1)>U(1)
$$

Lemma 2 implies $\tilde{q}(1)>q(1)$. From $(9)$ and $\eta=2$, we have $v(0)=$ $(1-\delta)^{-1}(r(0)+\theta(0))$. Lemma 2 implies $\tilde{v}(0)<v(0)$. 
Proof of Proposition 3: Suppose that $\delta=0$. Then

$$
\begin{aligned}
U(i) & =\lambda(i)-\lambda(j) \\
W(i) & =(\theta(i+1)-\theta(i))-(\theta(j+1)-\theta(j))
\end{aligned}
$$

that is, $\{U(i), W(i)\}$ are all constants. Lemma 1 then implies that there exists a unique solution to the equilibrium system. This proves equilibrium uniqueness for $\delta=0$.

By the same argument as in the proof of Lemma 2, there exists a finite $\bar{p}$ such that, if $p(i) \notin[-\bar{p}, \bar{p}]$, then the equilibrium equations fail to hold by an amount $\epsilon$ that is bounded away from zero. By continuity, if $\delta$ is in the neighborhood of $\delta=0$ there can be no solution to the system of equilibrium equations outside of $[-\bar{p}, \bar{p}]$. I thus henceforth restrict to this compact set of $p(i)$ values.

Consider the system of equations producing the equilibrium. This is given by the price equations (6), the quantity equations (3), the consumer value functions (4), and the firm value functions (9). Let

$$
\mathbf{x} \equiv(p(0), \ldots, p(\eta-1), q(0), \ldots, q(\eta-1), u(1), \ldots, u(\eta), v(0), \ldots, v(\eta-1))
$$

be the corresponding vector of equilibrium variables. We thus have a system of $4 \eta$ equations and $4 \eta$ unknowns. Represent this system as $f_{i}(\mathbf{x} ; \delta)=0$. The matrix of partial derivatives at $\delta=0, \nabla f$, is a block matrix:

$$
\nabla f=\left[\begin{array}{cccc}
I & I & 0 & 0 \\
A & I & B & 0 \\
0 & 0 & I & 0 \\
0 & I & 0 & I
\end{array}\right]
$$

where $A$ and $B$ have non-zero values in both diagonals and zero in every other position. It follows that $\nabla f$ has full rank. Since moreover all $f_{i}$ are continuously differentiable, the Implicit Function Theorem implies that there exists a unique equilibrium in the neighborhood of $\mathbf{x}^{*}$ and $\delta=0$, where $\mathbf{x}^{*}$ is the equilibrium at $\delta=0$. Finally, by continuity and the assumption that prices belong to a compact set, there exists no equilibrium outside the neighborhood of $\mathbf{x}^{*}$, which finally implies there exists a unique equilibrium in the neighborhood of $\delta=0$. This establishes the first part of the proposition.

I next turn to the equilibrium characterization. Consider first the case when $\theta(i+1)-\theta(i)$ is constant and Property 1 holds. If $\delta=0$, then $w(i)=$ 
$\theta(i+1)-\theta(i)$. It follows that $w(i)$ is constant and thus $W(i)=0$. Since $\lambda(i)>\lambda(i-1)$, it follows that $U(i)=U(i)+W(i)$ is strictly increasing, which by Lemma 2 implies that $x(i)$ is strictly decreasing. Finally, since $w(i)=\theta_{1}$, where $\theta_{1}$ is a constant, (6) reduces to

$$
p(i)=\frac{1-\Phi(x(i))}{\phi(x(i))}-\theta_{1},
$$

which, by part (iv) of Assumption 1, implies that $p(i)$ is strictly increasing in $i$.

Consider now the case when $\lambda(i)$ is constant and Property 2 holds. If $\delta=0$, then this implies that $u(i)=\lambda(i+1)$ is constant, and thus $U(i)=0$. Define $H^{\prime}(i) \equiv \partial H(i) / \partial x(i)$. Note that, by part (iv) of Assumption 1, $H^{\prime}(i)<0$; and that $\partial H(i) / \partial P(i)=H^{\prime}(i)$. Applying the Implicit Function Theorem to $(12)$ we get

$$
\frac{d P(i)}{d W(i)}=-\frac{1}{1-H^{\prime}(i)}<0 .
$$

Since $W(i)=w(i)-w(j)$, we have

$$
\frac{\partial P(i)}{\partial w(i)}=\frac{\partial P(i)}{\partial W(i)}
$$

Moreover, since

$$
h(i)=\frac{1-\Phi(P(i))}{\phi(P(i))}
$$

and

$$
H(i)=\frac{1-2 \Phi(P(i))}{\phi(P(i))}
$$

we have

$$
\frac{\partial h(i)}{\partial P(i)}=\frac{1}{2} \frac{\partial H(i)}{\partial P(i)}=\frac{1}{2} H^{\prime}(i) .
$$

Differentiating the right-hand side of (6) with respect to $w(i)$, we get

$$
\frac{d p(i)}{d w(i)}=\left(\frac{1}{2} H^{\prime}(i)\right)\left(-\frac{1}{1-H^{\prime}(i)}\right)-1<0 .
$$

If $\delta=0$, then $w(i)=\theta(i+1)-\theta(i)$, which is strictly increasing. It follows that $p(i)$ is strictly decreasing in $i$. Finally, the results follow from continuity 
in $\delta$.

Proof of Proposition 4: Equations (15) and Properties 1-2 imply that $U(i) \geq 0$ if and only if $i \geq i^{*}$, and likewise $W(i)>0$ if and only if $i \geq i^{*}$. Lemma 2 then implies that $q(i) \geq \frac{1}{2}$ if and only if $i \geq i^{*}$.

Suppose that $i^{*}<i<\eta-1$. From (10), the state moves away from $i^{*}$ in expected terms if and only if

$$
\frac{\eta-1-i}{\eta} q(i)>\frac{i}{\eta}(1-q(i))
$$

which is equivalent to

$$
q(i)>\frac{i}{\eta-1}
$$

In other words, the system moves away from $i^{*}$ if and only if the leader's birth rate, $q(i)$, is greater that the leader's death rate, $\frac{i}{\eta-1}$.

By the same argument as in the proof of Proposition 3, at $\delta=0$ and in the neighborhood of $\delta=0$ equilibrium prices are finite. This implies that $x(i)$ is bounded. Part (iii) of Assumption 1 then implies that $q(i)$ is bounded away from 1 . Therefore, at $i=\eta-1, q(i)<\frac{i}{\eta-1}$. Part (b) of the result follows.

Suppose now that $\lambda\left(i^{*}+1\right)-\lambda\left(i^{*}\right)>\lambda^{\prime}>0$ or $\theta\left(i^{*}+1\right)+\theta\left(i^{*}-1\right)-2 \theta\left(i^{*}\right)>$ $\theta^{\prime}>0$. Note that, by Property 1 , the first inequality implies $U(i)>0$ for all $i>i^{*}$; and, by Property 2, the second equality implies $W(i)>0$ for all $i>i^{*}$. By making $\lambda^{\prime}$ or $\theta^{\prime}$ large enough, I can make $U(i)+W(i)$ arbitrarily large. By Lemma 2, this implies I can make $q(i)$ arbitrarily close to 1 . The death rate, by contrast, is close to $\frac{1}{2}$. It follows that, for $i$ close to $i^{*},(16)$ holds true. Part (c) of the result follows.

Proof of Proposition 5: $\quad$ Let $\hat{y}$ be the derivative of a generic variable $y$ with respect to $\psi$ measured at $\psi=0$. Note that, at $\psi=0$, we have 
$q(i)=\bar{q}=\frac{1}{2}$ and $u(i)=\bar{u}$. Therefore, by differentiating (4), we get

$$
\begin{array}{r}
\hat{u}(i)=i+1+\delta\left(\frac{j}{\eta}(\hat{q}(i+1) \bar{u}+\bar{q} \hat{u}(i+2))+\left(\frac{j}{\eta} \hat{q}(j-1)+\frac{i}{\eta} \hat{q}(i)\right) \bar{u}+\right. \\
\left.\quad+\left(\frac{j}{\eta} \bar{q}+\frac{i}{\eta} \bar{q}\right) \hat{u}(i+1)+\frac{i}{\eta}(\hat{q}(j) \bar{u}+\bar{q} \hat{u}(i))\right), \\
=i+1+\delta \bar{u}\left(\frac{j}{\eta}(\hat{q}(i+1)+\hat{q}(j-1))+\frac{i}{\eta}(\hat{q}(i)+\hat{q}(j))\right)+ \\
\quad+\delta \bar{q}\left(\frac{j}{\eta} \hat{u}(i+2)+\frac{\eta-1}{\eta} \hat{u}(i+1)+\frac{i}{\eta} \hat{u}(i)\right) \\
=i+1+\delta \frac{1}{2}\left(\frac{j}{\eta} \hat{u}(i+2)+\frac{\eta-1}{\eta} \hat{u}(i+1)+\frac{i}{\eta} \hat{u}(i)\right)
\end{array}
$$

Subtracting by the corresponding expression for $j$, we have

$$
\hat{U}(i)=i-j+\frac{1}{2} \delta\left(\frac{j}{\eta} \hat{U}(i+1)+\frac{\eta-1}{\eta} \hat{U}(i)+\frac{i}{\eta} \hat{U}(i-1)\right)
$$

This system has an exact linear solution:

$$
\hat{U}(i)=\eta \frac{2 \psi i-(\eta-1) \psi}{\eta(1-\delta)+2 \delta}
$$

Since $\theta(i)=0$, the firm value function is given by

$$
v(i)=r(i)+\delta\left(\frac{j+1}{\eta} v(i)+\frac{i}{\eta} v(i-1)\right)
$$

where

$$
\begin{aligned}
r(i) & =\frac{q(i)^{2}}{-q^{\prime}(i)} \\
q(i) & =1-\Phi(P(i)-U(i))
\end{aligned}
$$

Taking into account that, at $\psi=0, q(i)=\bar{q}=\frac{1}{2}$; and that $\phi^{\prime}(0)=0$; it follows that

$$
\hat{r}(i)=\frac{2 \bar{q}(-\phi(0))(\hat{P}(i)-\hat{U}(i)) \phi(0)}{\phi(0)^{2}}=\hat{U}(i)-\hat{P}(i)
$$


Taking derivatives of (17) with respect to $\psi$ at $\psi=0$, we get

$$
\hat{v}(i)=\hat{U}(i)-\hat{P}(i)+\delta\left(\frac{j+1}{\eta} \hat{v}(i)+\frac{i}{\eta} \hat{v}(i-1)\right)
$$

Since $j=\eta-1-i$, this can be rearranged as

$$
(1-\delta) \hat{v}(i)=\hat{U}(i)-\hat{P}(i)-\delta \frac{i}{\eta}(\hat{v}(i)-\hat{v}(i-1))
$$

Likewise, for firm $j$,

$$
(1-\delta) \hat{v}(j)=\hat{U}(j)-\hat{P}(j)-\delta \frac{j}{\eta}(\hat{v}(j)-\hat{v}(j-1))
$$

Since $U(j)=-U(i)$ and $P(j)=-P(i)$, we can add the above equations to get

$$
(1-\delta)(\hat{v}(i)+\hat{v}(j))=-\delta \frac{i}{\eta}(\hat{v}(i)-\hat{v}(i-1))-\delta \frac{j}{\eta}(\hat{v}(j)-\hat{v}(j-1))
$$

which has a linear solution (notice that a linear $\hat{v}$ implies that both the left-hand side and the right-hand side are constant in $i, j$ ).

The price equation is given by

$$
p(i)=h(i)-\delta \frac{i}{\eta}(v(i+1)-v(i))-\delta \frac{j}{\eta}(v(i)-v(i-1))
$$

where

$$
\begin{aligned}
h(i) & =\frac{q(i)}{-q^{\prime}(i)} \\
q(i) & =1-\Phi(P(i)-U(i))
\end{aligned}
$$

Taking into account that, at $\psi=0, q(i)=\bar{q}=\frac{1}{2}$; and that $\phi^{\prime}(0)=0$; it follows that

$$
\hat{h}(i)=\frac{-\phi(0)(\hat{P}(i)-\hat{U}(i)) \phi(0)}{\phi(0)^{2}}=\hat{U}(i)-\hat{P}(i)
$$

Taking derivatives of (19) with respect to $\psi$ at $\psi=0$, we get

$$
\hat{p}(i)=\hat{U}(i)-\hat{P}(i)-\delta \frac{i}{\eta}(\hat{v}(i+1)-\hat{v}(i))-\delta \frac{j}{\eta}(\hat{v}(i)-\hat{v}(i-1)),
$$


Subtracting the $\hat{p}(j)$ equation from the $\hat{p}(i)$ equation, and taking into account that $\hat{v}(i)$ is linear, we have

$$
\hat{P}(i)=2 \hat{U}(i)-2 \hat{P}(i)
$$

or simply

$$
\hat{P}(i)=\frac{2}{3} \hat{U}(i)
$$

Plugging this back into the price function, we get

$$
\hat{p}(i)=\frac{1}{3} \hat{U}(i)-\kappa,
$$

where $\kappa$ is a constant (since $v(i)$ is linear and $i+j=\eta-1$ ). Since $\hat{U}(i)$ is increasing, it follows that $\hat{p}(i)$ is increasing. This in turn implies part (a) of the proposition.

Since

$$
q(i)=1-\Phi(P(i)-U(i))
$$

it follows, by (20), that

$$
\hat{q}(i)=-\phi(0)(\hat{P}(i)-\hat{U}(i))=\frac{\phi(0)}{3} \hat{U}(i)
$$

Since $\hat{U}(i)$ is increasing, so is $\hat{q}(i)$, which proves part (b) of the proposition.

Part (c) is fairly straightforward: since $\psi$ is small, $q(i)$, the birth rate, is approximately constant. The death rate, however, is given by $i / \eta$.

Plugging (20) into the value function, we get

$$
(1-\delta) \hat{v}(i)=\frac{1}{3} \hat{U}(i)-\delta \frac{i}{\eta}(\hat{v}(i)-\hat{v}(i-1))
$$

Since $\hat{v}$ is linear and $U$ is increasing, the right-hand side is increasing in $i$. It follows that $\hat{v}$ is also increasing, which in turn implies part (d) of the proposition.

Finally, the fact that $v$ is increasing implies that the left-hand side of (18) is negative, which in turn implies part (e) of the proposition.

Derivation of $\theta(i)$ and $\lambda(i)$ in wireless telecommunications example. Utility per consumer pair is given by

$$
u_{C}\left(q_{C}\right)=\left(\omega-\frac{1}{2} q_{C}\right) q_{C}
$$


This leads to a demand curve $q_{C}=\omega-p_{C}$. Firm profit (for a pair of users) is given by

$$
\pi=\left(p_{C}-c\right) q_{C}=\left(\omega-q_{C}-c\right) q_{C}
$$

where $c$ is the cost of a call. Optimal output and price are therefore given by

$$
\begin{aligned}
& q_{C}^{*}=\frac{\omega-c}{2} \\
& p_{C}^{*}=\frac{\omega+c}{2}
\end{aligned}
$$

Substituting (24) for $q_{C}$ in (22) and (23), and subtracting expenditure from gross surplus, I obtain equilibrium net consumer surplus and profit:

$$
\begin{aligned}
\mu^{*} & =\frac{1}{2}\left(\frac{\omega-c}{2}\right)^{2} \\
\pi^{*} & =\left(\frac{\omega-c}{2}\right)^{2}
\end{aligned}
$$

I now apply these generic expressions to the particular values of cost of onnet and off-net calls. Specifically, profit per on-net caller pair and profit per off-net caller pair are respectively given by

$$
\begin{aligned}
& \tilde{\pi}(i)=\left(\frac{\omega-2 c_{0}-c_{1}}{2}\right)^{2} \\
& \hat{\pi}(i)=\left(\frac{\omega-c_{0}-c_{1}-a(j)}{2}\right)^{2}
\end{aligned}
$$

Finally, the number of off-net calls originating in network $j$, per caller pair, is given by

$$
\hat{q}_{C}(j)=\frac{\omega-c_{0}-c_{1}-a(i)}{2} .
$$

Pulling all of these expressions together, I get the following equilibrium profit function:

$$
\begin{array}{r}
\theta(i)=i(i-1) \tilde{\pi}(i)+i j \hat{\pi}(i)+i j \hat{q}(j)\left(a(i)-c_{0}\right) \\
=i(i-1)\left(\frac{\omega-2 c_{0}-c_{1}}{2}\right)^{2}+i j\left(\frac{\omega-c_{0}-c_{1}-a(j)}{2}\right)^{2}+ \\
\quad+i j\left(\frac{\omega-c_{0}-c_{1}-a(i)}{2}\right)\left(a(i)-c_{0}\right)
\end{array}
$$


The first term corresponds to calls from a consumer to the other consumers in the same network. The second term corresponds to calls from a consumer to consumers in the other network. Finally, the third term corresponds to revenues from charging termination fees to the other network.

As to the consumer net utility function, we have

$$
\lambda(i)=(i-1) \frac{1}{2}\left(\frac{\omega-2 c_{0}-c_{1}}{2}\right)^{2}+j \frac{1}{2}\left(\frac{\omega-c_{0}-c_{1}-a(j)}{2}\right)^{2}
$$

Again, the first term corresponds to on-net calls and the second one to off-net calls. Notice that, if $a(j)>c_{0}$, then the coefficient on $(i-1)$ is greater than the coefficient on $j$. It follows that $\lambda(i)$ is strictly increasing in $i$, that is, Property 1 holds. 


\section{References}

Arthur, W Brian (1989), "Competing technologies, increasing returns, and lock-in by historical events," Economic Journal 99, 116-131.

Athey, Susan, And Armin Schmutzler (2001), "Investment and Market Dominance," RAND Journal of Economics 32, 1-26.

Auriol, Emmanuelle, and Michel Benaim (2000), "Standardization in Decentralized Economies," American Economic Review 90, 550-570.

Bagnoli, Mark, and Theodore Bergstrom (2005), "Log Concave Probability and Its Applications," Economic Theory 26, 445-469.

Beggs, Alan W, and Paul Klemperer (1992), "Multi-period Competition with Switching Costs," Econometrica 60, 651-666.

Budd, Christopher, Christopher Harris and John Vickers (1993), "A Model of the Evolution of Duopoly: Does the Asymmetry Between Firms Tend to Increase or Decrease?," Review of Economic Studies 60, 543-573.

CABral, Luís (2000), Introduction to Industrial Organization, Cambridge, MA: MIT Press.

CABral, Luís (2002), "Increasing Dominance With no Efficiency Effect," Journal of Economic Theory 102, 471-479.

Cabral, Luís, and Michael H Riordan (1994), "The Learning Curve, Market Dominance and Predatory Pricing," Econometrica 62, 1115-1140.

Cabral, Luís M B, and J Miguel Villas-Boas (2005), "Bertrand Supertraps," Management Science 51, 599-613.

Caplin, Andrew, and Barry Nalebuff (1991), "Aggregation and Imperfect Competition: On the Existence of Equilibrium," Econometrica 59, 25-59.

Doganoglu, Toker (2003), "Dynamic Price Competition with Consumption Externalities," Netnomics 5, 43-69.

Doraszelski, Ulrich, Jiawei Chen, And Joseph Harrington (2007), “Avoiding Market Dominance: Product Compatibility in Markets with Network Effects," Harvard University, University of California-Irvine, and Johns Hopkins University. 
Doraszelski, Ulrich, and Ariel Pakes (2007), "A Framework for Applied Dynamic Analysis in IO," in Mark Armstrong and Robert Porter (eds.), Handbook of Industrial Organization, Volume 3, North-Holland, Amsterdam.

Doraszelski, Ulrich, And Mark Satterthwaite (2007), "Computable MarkovPerfect Industry Dynamics: Existence, Purification, and Multiplicity," Harvard and northweestern Universities.

Driskill, Robert (2007), "Monopoly and Oligopoly Supply of a Good with Dynamic Network Externalities," Vanderbilt University.

Economides, Nicholas (1996), "The Economics of Networks," International Journal of Industrial Organization 14, 673-699.

Farrell, Joseph, and Paul Klemperer (2007), "Coordination and Lock-In: Competition with Switching Costs and Network Effects," in M Armstrong and R Porter (Eds), Handbook of Industrial Organization, Vol 3, NorthHolland.

Farrell, Joe, and Garth Saloner (1985), "Standardization, compatibility and innovation," Rand Journal of Economics 16, 70-83.

Fudenberg, Drew, And Jean Tirole (2000), "Pricing Under the Threat of Entry by a Sole Supplier of a Network Good," Journal of Industrial Economics 48, 373-390.

Gandal, Neil (1994), "Hedonic price indexes for spreadsheets and an empirical test for Network externalities," Rand Journal of Economics 25, 160-170.

Gilbert, Richard J., And David M. G. newbery (1982), "Preemptive Patenting and the Persistence of Monopoly Power," American Economic Review 72, 514-526.

Goolsbee, Austen, And Peter J Klenow (2002), "Evidence on learning and Network externalities in the diffusion of home computers," Journal of Law and Economics 45, 317-344.

Calzada, J, and T. Valletti (2008), "Network Competition and Entry Deterrence," Economics Journal 118, 1223-1244.

European Regulatory Group (2008), "ERGs Common Position on Symmetry of Fixed Call Termination Rates and Symmetry of Mobile Call Termination Rates," CP adopted by the ERG-Plenary on 28th February 2008. 
Harbord, David, And Marco Pagnozzi (2008), "On-Net/Off-Net Price Discrimination and 'Bill-and-Keep' vs. 'Cost-Based' Regulation of Mobile Termination Rates," Market Analysis and Università di Napoli Federico II.

Kandori Michiniro, and Rafael Rob (1998), "Bandwagon Effects and Long Run Technology Choice Pages," Games and Economic Behavior 22, 30-60.

Katz, Michal L, And Carl Shapiro (1985), "Network externalities, competition and compatibility," American Economic Review 75, 424-440.

Laffont, Jean-Jacques, Patrick Rey, and Jean Tirole (1998a), "Network Competition: I. Overview and nondiscriminatory Pricing," RAND Journal of Economics 29, 1-37.

Laffont, Jean-Jacques, Patrick Rey, and Jean Tirole (1998b), "Network Competition: II. Price Discrimination," RAND Journal of Economics 29, $38-56$.

Laussel, Didier, and Joana Resende (2007), "Competition in the Market vs Competition for the Market: The Case of Cell Phone Networks," GREQAM and CORE, November.

Llobet, Gerard, and Michael Manove (2006), "Network size and Network capture," Working Paper, CEMFI and Boston University.

Markovich, SARIT (2004), "Snowball: A Dynamic Oligopoly Model with Network Externalities," Northwestern University.

Markovich, Sarit, and Johannes Moenius (2004), "Winning While Losing: Competition Dynamics in the Presence of Indirect Network Effects," Northwestern University.

Mitchell, Matthew, And Andrzej Skrzypacz (2006), "Network Externalities and Long-Run Market Share," Economic Theory 29, 621-648.

Murkres, James R. (1991), Analysis on Manifolds, Menlo Park, CA: AddisonWesley Publishing Company.

Peitz, Martin (2005), "Asymmetric Regulation of Access and Price Discrimination in Telecommunications," Journal of Regulatory Economics 28, 327-343.

Reinganum, Jennifer F. (1982), "Uncertain Innovation and the Persistence of Monopoly," American Economic Review 73, 741-748. 
Rysman, Marc (2004), "Competition Between Networks: a Study of the Market for Yellow Pages," The Review of Economic Studies 71, 483-512.

Vodafone (2008), "ERG Public Consultation on a Draft Common Position on Symmetry of Mobile/Fixed Call Termination Rates," January. 